\title{
Endoscopic endonasal management of non-functioning pituitary adenomas with cavernous sinus invasion: a 10-
} year experience*

\author{
Fabio Ferreli', Mario Turri-Zanoni', Frank Rikki Canevari², Paolo Battaglia', \\ Maurizio Bignami', Paolo Castelnuovo', Davide Locatelli³ \\ Rhinology 53: 308-316, 2015 \\ DOI:10.4193/Rhino14.309 \\ ' Department of Otorhinolaryngology, University of Insubria, Varese, Italy \\ *Received for publication: \\ 2 Department of Otorhinolaryngology, SS Antonio Biagio e Cesare Arrigo Hospital of Alessandria, Italy \\ December 15, 2014 \\ ${ }^{3}$ Department of Neurosurgery, Civic Hospital, Legnano, Italy \\ Accepted: May 8, 2015
}

\begin{abstract}
Background: The management of Non-Functioning Pituitary Adenoma (NFPA) invading the cavernous sinus (CS) is currently a balancing act between the surgical decompression of neural structures, radiotherapy and a wait-and-see policy.

Methods: We undertook a retrospective review of 56 cases of NFPA with CS invasion treated through an endoscopic endonasal approach (EEA) between 2000 and 2010. The Knosp classification was adopted to describe CS involvement using information from preoperative MRI and intraoperative findings. Extent of resection and surgical outcomes were evaluated on the basis of postoperative contrast-enhanced MRI. Endocrinological improvement and visual outcomes were assessed according to the most recent consensus criteria.
\end{abstract}

Results: EEA was performed using direct para-septal, trans-ethmoidal-sphenoidal or trans-ethmoidal-pterygoidal-sphenoidal approach. Visual outcomes improved in 30 (81\%) patients. Normalization or at least improvement of previous hypopituitarism was obtained in $55 \%$ of cases. A gross total resection was achieved in $30.3 \%$ of cases. The recurrence-free survival was $87.5 \%$, with a mean follow-up of 61 months (range, 36-166 months). No major intraoperative or postoperative complications occurred.

Discussion: EEA is a minimally-invasive, safe and effective procedure for the management of NFPA invading the CS. The extent of CS involvement was the main factor limiting the degree of tumor resection. The EEA was able to resolve the mass effect, preserving or restoring visual function, and obtaining adequate long-term tumor control.

Key words: cavernous sinus, endoscopic endonasal, pituitary adenoma, skull base, trans-sphenoidal surgery

\section{Introduction}

Non-functioning pituitary adenomas (NFPA) are pituitary tumours without hormonal hyperproduction but which create a mass effect leading to visual defects and which may progress to hypopituitarism. Immunonegative, gonadotropic, and silent adenomas are grouped under the name of NFPA since they have no specific clinical symptoms of hormone hypersecretion ${ }^{(1-3)}$. Trans-sphenoidal surgery is considered the treatment of choice, given the lack of efficacy of the standard medical treatment in reducing the size of NFPA. These lesions are usually diagnosed when they have grown to large sizes (macroadenoma, greater than $1 \mathrm{~cm}$ ), and they remain a challenge for skull base surgeons given the frequent occurrence of residual tumor ${ }^{(4)}$. Most recently, surgical resection of pituitary macroadenomas has been facilited by the use of an angled endoscope in combination with extended endoscopic endonasal approaches (EEA) ${ }^{(5-8)}$. However, once the tumor has significantly invaded the cavernous sinus (CS), a gross total resection (GTR) is achievable only in a small percentage of cases because of the large dimension of lesions and their proximity to critical neurovascular structures. The surgical purposes in these cases are to obtain the maximal tumor resection, to improve visual function and neurological symptoms whilst at the same time preserving the hormonal function of the healthy pituitary gland. In the present 
study we describe our experience of using an EEA for the surgical management of NFPA invading the CS and we evaluate the long-term results of a wait-and-see policy after surgery for residual tumors.

\section{Methods}

After approval by the Institutional Review Board we conducted a retrospective analysis of 205 endoscopic endonasal trans-sphenoidal procedures for NFPA performed at the University Hospital of Pavia and at the University Hospital of Insubria-Varese between 2000 and 2010. Among these, only patients who presented with CS invasion (CSI) both on preoperative imaging and intraoperatively were included in this study. Preoperative evaluation with 1.5-T contrast-enhanced MR imaging was performed for all patients and the imaging findings were evaluated using the grading system proposed by Knosp to predict the probability of CSI (grade 3 and 4) ${ }^{(9)}$. Exclusion criteria were as follows: grade 1 and 2 according to Knosp; patients who had previously been treated with radiotherapy in the pituitary region; patients with follow-up shorter than 36 months. All patients enrolled underwent neurological, ophthalmological and endocrinological examinations before and after surgery. All the patients gave their informed consent to participate in this survey.

\section{Neuroradiological assessment}

Paranasal sinus computed tomography (CT) scans were performed for all patients in order to evaluate anatomical variants in the sellar floor, nasal cavity and paranasal sinus. All patients also underwent tumor evaluation by magnetic resonance imaging (MRI). Pre-surgical imaging protocol included systematic T1weighted sequence with and without contrast injection and T2 sequence. Tumor volume was estimated using a modified ellipsoid volume formula (length $\mathrm{x}$ height $\mathrm{x}$ width $\mathrm{x}$ [pi/6]), based on MR imaging. Tumor lateral extension, particularly focusing on CSI, was defined using coronal T2 and T1-weighted. At three, six and twelve months after surgery and then once annually, all patients underwent postoperative MR imaging control with the same sequences as those performed preoperatively.

The first MR scan, performed on the 3rd postoperative month, was used to assess the degree of tumor resection. Absence of tumor was considered as GTR (Figure 2). If present, the volume of residual adenomas was calculated with the same mathematical formula used for the initial tumor measurement. The residual tumor volume was compared to the preoperative tumor volume thus obtaining the percentage of tumor resection which was classified as follows: subtotal removal (STR), with a residual lesion less than 20\% (Figure 3); partial removal (PARTIAL), with residual lesion less than 50\% (Figure 4). Preoperative and postoperative radiological evaluations were performed by an independent neuroradiologist in all cases.

\section{Endocrinological assessment}

Before and after surgery, all patients underwent endocrinological tests to evaluate the anterior pituitary function. Preoperative pituitary endocrine function laboratory data were collected for all patients and included follicle-stimulating hormone, thyroid-stimulating hormone, T3, T4, cortisol, adrenocorticotropic hormone, insulin-like growth factor-1 and prolactin levels. Endocrine function was assessed with provocative or dynamic test. The same endocrinological tests were repeated immediately post-operatively, at three, six and twelve months after surgery and thereafter once a year. Post-operative findings were classified as improved or worsened when a given component of the pituitary function had been recovered or lost, and stable if no change occurred.

\section{Ophthalmological examination}

A designated neuro-ophthalmologist interpreted the visual field (VF) examination (Humphrey and/or Goldmann test) and assessed visual acuity (VA), color acuity, ocular movements (III, IV and VI cranial nerve function) and fundoscopic examination preoperatively, immediately post-operatively and at three, six and twelve months following surgery and afterwards once a year. The visual outcomes were classified as follows: normal, when all parameters were normalized; improved, when at least one of the parameters had been improved; stable, when no change occurred; worsened, when at least one of the parameters showed deterioration.

\section{Surgical approach}

The endoscopic endonasal approach to the sellar region used by our group has been previously published ${ }^{(10)}$. All the procedures were performed through the binostril approach. The endoscopic endonasal approach to the sphenoid and sellar compartment was tailored to the tumor extension according to three different techniques: direct paraseptal approach (DP), trans-ethmoidsphenoid approach (TES) or trans-ethmoid-pterygoid-sphenoid approach (TEPS). The indication for the DP approach relates to lesions localized in the sellar region or extending to the medial part of the CS. The indication for the TES approach is when there is lateral and anterior extension of the pituitary tumor. The TEPS approach is indicated when the tumor has spread to the lateral part of the CS, middle cranial fossa and infratemporal fossa. A wide bilateral sphenoidotomy with removal of $0.5-1 \mathrm{~cm}$ of posterior nasal septum is always performed to allow a two nostril four-hands technique. Importantly, before the sphenoidotomy, we strongly advise detaching the mucosal layer of the sphenoidal floor in order to identify and preserve the choanal branch of the sphenopalatine artery (nasoseptal rescue flap). The approach to the sellar region is similar in all the three techniques and requires the identification of critical orientating anatomical landmarks utilising intraoperative Doppler Ultra- 


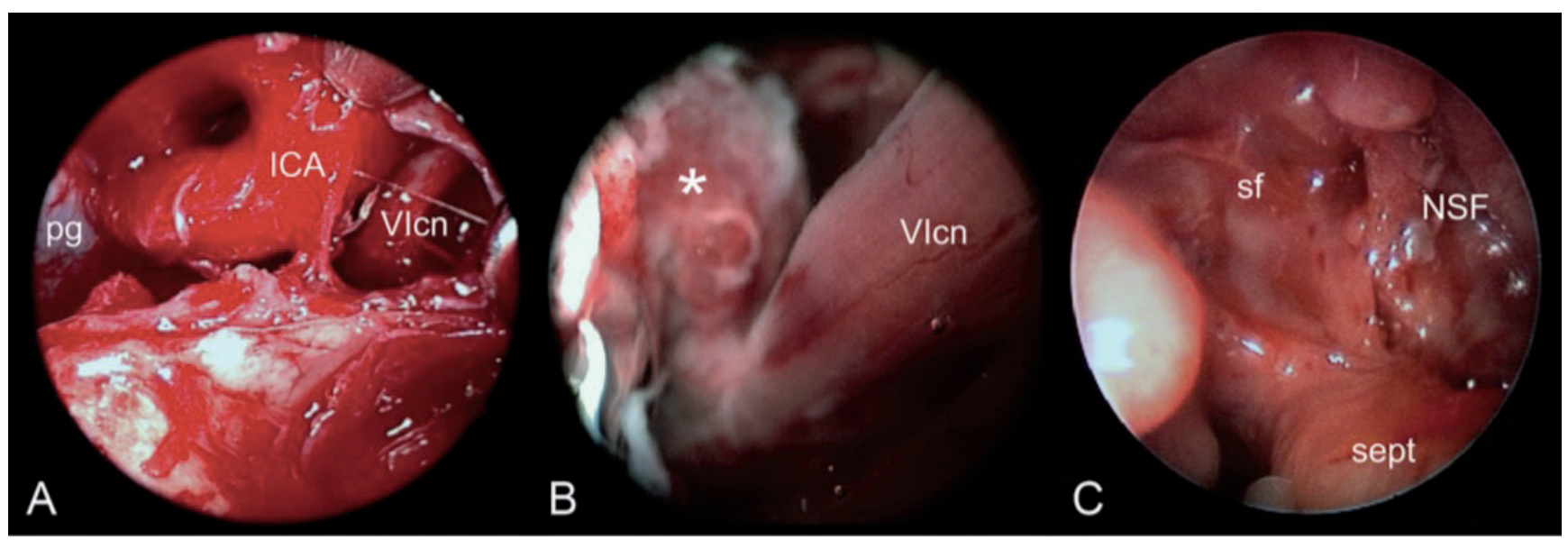

Figure 1. Intraoperative view through a $0^{\circ}$ endoscope. A) After opening the dura of the cavernous sinus, the internal carotid artery and the sixth cranial nerve were exposed and the pituitary adenoma invading the cavernous sinus was removed. B) The 'diving technique' allowed us to explore the cavernous sinus and it was useful in identifying a small residual tumor (marked with a white asterisk) that had to be removed in order to obtain a gross-total resection. C) Post-operative endoscopic endonasal control three months after surgery, showing the integration of the nasoseptal flap with complete healing of the surgical cavity. Labelled structures: pg, pituitary gland; ICA, internal carotid artery; VIcn, sixth cranial nerve; sf, sella floor; sept, nasal septum; NSF, nasoseptal flap.

sound and neuronavigation systems. Lateral tumor extension in the parasellar region requires removal of the bony wall covering the cavernous part of the ICA and the postero-lateral wall of the sphenoid extending up to the orbital apex. Once a window in the bone of the sellar floor is opened, the procedure is carried out with the incision of the sellar dural layer and subsequent tumor debulking. The continuous flushing and the hydrodissection of the sellar cavity ("diving surgery") is useful in identifying small infiltrations of the CS and checking the integrity of the pituitary stalk when the endoscope is introduced into the sellar compartment (Figure 1) ${ }^{(11)}$. In most cases, when tumour removal is completed reconstruction of the sellar floor is not required. In cases presenting with a cerebrospinal fluid (CSF) leak, closure is performed using the nasoseptal flap overlay combined with autologous tissue graft placed inlay ${ }^{(12-14)}$.

\section{Results}

Of the 205 patients treated for a NFPA, 56 (27.3\%) were affected by tumors with CSI (grade 3-4 according to Knosp). This group comprised 20 males and 36 females. The patients were aged between 37 and 79 with a mean age of 59 years. Clinical presentations necessitating surgery included visual disturbances (37.5\%), headache (19.6\%) and symptoms related to hypopituitarism (17.8\%). The preoperative ophthalmologic assessment and the endocrinological data are reported in Table 1.

\section{Neuroimaging}

On examination of the radiological findings from MRI scans, 34 cases $(60.7 \%)$ were classified as macroadenoma $(>1 \mathrm{~cm})$, while 22 cases $(39.3 \%)$ as giant adenoma $(>4 \mathrm{~cm})$. The average pre- operative tumor volume was $19.7 \mathrm{~cm}^{3}$ ranging between 4.3 and $73.9 \mathrm{~cm}^{3}$. The parasellar extension of the tumor was classified using the Knosp grading system as follows: $28 / 56$ (50\%) cases were classified with a grade 3 and 28/56 (50\%) cases with a grade 4 . In all cases the CSI was confirmed by the intraoperative findings as well (Table 1).
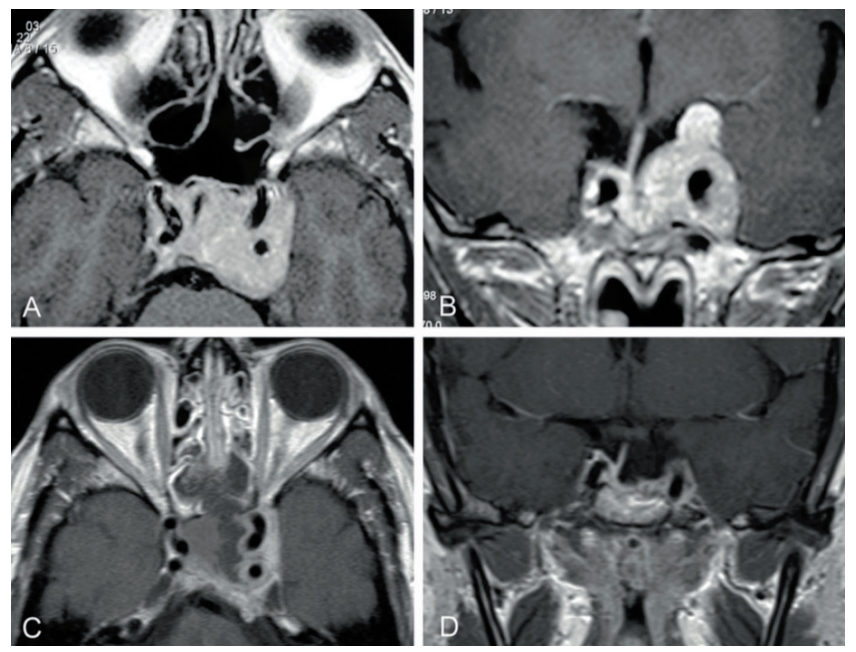

Figure 2. Preoperative contrast-enhanced T1-weighted MRI scan in axial (A) and coronal (B) view, showing a non-functioning pituitary adenoma invading the left cavernous sinus with encasement of the internal carotid artery. The 1 year postoperative contrast-enhanced T1-weighted MRI scan in axial (C) and coronal (D) view revealed a gross-total resection of the lesion. 
Table 1. Summary of the clinical and pathological findings of the patients enrolled in this survey.

\begin{tabular}{|c|c|}
\hline NFPA with Cavernous Sinus Invasion & Number \\
\hline Total of patients (\%) & $\begin{array}{c}56(27.3 \% \text { of NFPA } \\
\text { treated })\end{array}$ \\
\hline \multicolumn{2}{|l|}{ Sex } \\
\hline Female & 20 \\
\hline Male & 36 \\
\hline \multicolumn{2}{|l|}{ Age } \\
\hline Median & 59 \\
\hline Range & $37-79$ \\
\hline \multicolumn{2}{|l|}{ Surgical indications } \\
\hline Incidental findings & $8(14 \%)$ \\
\hline Residual progression & $8(14 \%)$ \\
\hline Endocrinological disorders & $10(17.8 \%)$ \\
\hline Visual deficit & $21(37.5 \%)$ \\
\hline Headache & $11(19.6 \%)$ \\
\hline \multicolumn{2}{|l|}{ Clinical examination } \\
\hline \multicolumn{2}{|l|}{ Ophthalmology } \\
\hline Normal & $19(33.9 \%)$ \\
\hline Decrease in VA & $4(7.1 \%)$ \\
\hline Abnormal VF & $16(28.5 \%)$ \\
\hline Abnormal VA \& VF & $15(26.7 \%)$ \\
\hline Palsy of III CN & $1(1.7 \%)$ \\
\hline Abnormal VA \& VF \& III CN palsy & $1(1.7 \%)$ \\
\hline \multicolumn{2}{|l|}{ Endocrinology } \\
\hline Normal pituitary function & $33(58.9 \%)$ \\
\hline Partial or hypopituitarism & $20(35.7 \%)$ \\
\hline Complete loss of pituitary function & $3(5.4 \%)$ \\
\hline \multicolumn{2}{|l|}{ Tumor size (\%) } \\
\hline $\operatorname{Macro}(>1 \mathrm{~cm})$ & $34(60.7 \%)$ \\
\hline Giant (>4cm) & $22(39.3 \%)$ \\
\hline \multicolumn{2}{|l|}{ Knosp classification (\%) } \\
\hline Grade 3 & $28(50 \%)$ \\
\hline Grade 4 & $28(50 \%)$ \\
\hline
\end{tabular}

Abbreviations. NFPA, non-functioning pituitary adenoma; VA, visual acuity; VF, visual field; CN, cranial nerve.

\section{Surgical treatment}

The DP approach was used in 35/56 patients (62.5\%), and included 32/34 macroadenomas (94.1\%) and 13/22 giant adenomas
Table 2. Visual outcomes overview comparing the pre-operative condition with the post-operative results.

\begin{tabular}{|c|c|c|c|c|c|}
\hline Pre-op & Post-op & Number & GTR & STR & PR \\
\hline \multirow[t]{3}{*}{ Normal vision } & & $19(33,9 \%)$ & & & \\
\hline & Stable & $100 \%$ & 5 & 12 & 2 \\
\hline & Worsened & $0 \%$ & 0 & 0 & 0 \\
\hline \multirow[t]{6}{*}{ Visual impairment } & & 37 (66.1\%) & & & \\
\hline & Worsened & $1(2.7 \%)$ & 0 & 1 & 0 \\
\hline & Stable & $6(16.2 \%)$ & 1 & 4 & 1 \\
\hline & $\begin{array}{c}\text { Total } \\
\text { improvement }\end{array}$ & 30 (81\%) & & & \\
\hline & Improved & $16(43.2 \%)$ & 4 & 10 & 2 \\
\hline & Normalized & $14(37.8 \%)$ & 7 & 7 & 0 \\
\hline
\end{tabular}

Abbreviations. GTR, gross total resection; STR, subtotal resection; PR, partial resection.

(59\%) with Knosp grade 3 in 23/28 cases, (82.1\%) and grade 4 in 12/28 cases, (42.8\%). The TES approach was performed in $17 / 56$ patients (30.3\%), including $2 / 34$ macroadenomas $(5.8 \%)$ and 5/22 giant adenomas (22.7\%), with Knosp grade 3 in 4/28 tumors, (14.2\%) and grade 4 in 13/28 tumors, (46.4\%). The TEPS approach was used in $4 / 56$ patients (7.2\%) affected by giant adenomas $4 / 22$ cases ( $18.1 \%)$; one case $(1 / 28,3.5 \%)$ was classified as Knosp grade 3 and the remaining three $(3 / 28,10.7 \%)$ as Knosp grade 4. GTR was achieved in 17/56 (30.3\%) patients, $12 / 28$ cases of Knosp grade 3 tumors, (42.8\%) and 5/28 cases of Knosp grade 4 tumors, (17.8\%); STR in 34/56 patients (60.7\%), while a PARTIAL in 5/56 patients (8.9\%). No recurrences were observed after GTR. Of the 39 patients with residual tumor, $7 / 39$ (17.9\%) required further surgical treatment via endoscopic transnasal approach for regrowth of the residual lesion.

\section{Clinical follow-up}

The mean follow-up period was 61 months with a range of 36166 months.

None of the 19 patients (33.9\%) with normal eyesight prior to surgery developed any deficit post-operatively. Of the 37 patients (66.1\%) presenting with visual defects $81 \%$ improved or normalized their visual function, $16.2 \%$ remained stable and one patient (2.7\%) experienced a worsening of his pre-existing deficit (Table 2).

From an endocrinological viewpoint information was available for only 44/56 patients as the remaining 12 had been referred to us solely for their surgery and their endocrinological follow up 
Table 3. Endocrinological outcomes overview comparing the preoperative condition with the post-operative results (data available for 44 patients).

\begin{tabular}{|c|c|c|c|c|c|}
\hline Pre-op & Post-op & Number & GTR & STR & PR \\
\hline \multirow[t]{3}{*}{ Normal (\%) } & & $21(47,7 \%)$ & 6 & 12 & 3 \\
\hline & No changes & $17(80,9 \%)$ & 6 & 9 & 2 \\
\hline & Worsened & $4(19,1 \%)$ & 0 & 3 & 1 \\
\hline \multirow[t]{5}{*}{ Hypopituitarism } & & $20(45,5 \%)$ & 7 & 12 & 1 \\
\hline & Worsened & $2(10 \%)$ & 2 & 0 & 0 \\
\hline & No changes & 7 (35\%) & 2 & 4 & 1 \\
\hline & Improved & $6(30 \%)$ & 1 & 5 & 0 \\
\hline & Normalized & $5(25 \%)$ & 2 & 3 & 0 \\
\hline \multirow[t]{4}{*}{ Panhypopituitarism } & & $3(6,8 \%)$ & 1 & 1 & 1 \\
\hline & No changes & $2(66,6 \%)$ & 0 & 1 & 1 \\
\hline & Improved & $1(33,3 \%)$ & 1 & 0 & 0 \\
\hline & Normalized & $0 \%$ & 0 & 0 & 0 \\
\hline
\end{tabular}

Abbreviations. GTR, gross total resection; STR, subtotal resection; PR, partial resection.

was undertaken in other centers. Of the patients with normal anterior pituitary function before surgery (21/44 patients, $47.7 \%)$, post-operative worsening occurred in only four cases (19.1\%) and for the remaining 17 (80.9\%) no variation in endocrine function was registered. For those patients presenting with partial pituitary deficiency, endoscopic surgery enabled a total or partial recovery of pituitary function in $55 \%$ of cases. Only one patient who had presented with total anterior pituitary deficit improved after surgery (Table 3).

Overall the pituitary axes affected by a functional deficit during the post-operative period were as follows: Growth Hormone deficiency was observed in 19 (43.1\%) patients; hypogonadism in 13 (29.5\%) cases; hypoprolactinemia in $6(13.6 \%)$ patients; hypocortisolism was detected in $9(20.4 \%)$ patients on postoperative day 2 increasing to 14 (31.8\%) patients on postoperative day 6; hypothyroidism occurred in 15 (34.1\%) cases. Patients with abnormal serum cortisol levels were treated with glucocorticoid replacement. Patients with postoperative hypothyroidism were subsequently treated with a levothyroxine replacement.

\section{Complications}

No perioperative mortality occurred in this cohort. Out of the six patients with intraoperative evidence of CSF-leak (10.6\%),
Table 4. Analysis of complications occurring in our series of 56 NFPA with CSI treated through an endoscopic transnasal trans-sphenoidal approach.

\begin{tabular}{|lcccc|}
\hline Complications & Total & GTR & STR & PR \\
\hline Post-operative CSF leak & $3(5.3 \%)$ & 0 & 3 & 0 \\
\hline Post-operative epistaxis & 0 & 0 & 0 & 0 \\
\hline Brain lesion/Hematoma & 0 & 0 & 0 & 0 \\
\hline III c.n. deficit & 0 & 0 & 0 & 0 \\
\hline VI c.n. transient defict & $1(1.7 \%)$ & 0 & 1 & 0 \\
\hline Internal carotid artery lesion & 0 & 0 & 0 & 0 \\
\hline Transient diabetes insipidus & $1(1.7 \%)$ & 1 & 0 & 0 \\
\hline Permanent diabetes insipidus & $2(3.5 \%)$ & 0 & 1 & 1 \\
\hline Death & 0 & 0 & 0 & 0 \\
\hline Meningitis & 0 & 0 & 0 & 0 \\
\hline
\end{tabular}

Abbreviations. GTR, gross total resection; STR, subtotal resection; PR, partial resection, c.n., cranial nerve.

four cases (who were treated before 2008) underwent sellar floor reconstruction using autologous tissue grafts (fascia lata, fat tissue, cartilage). Of these a post-operative CSF-leak occurred in three out of the four cases and required a revision surgery to obtain long-term closure. The remaining two patients with intraoperative evidence of CSF-leak who were treated after 2008 underwent closure with autologous tissue graft placed inlay covered by a nasoseptal flap placed overlay, obtaining effective long-term closure in both cases. Significantly, no postoperative CSF leak occurred in patients with no intraoperative evidence of CSF-leak.

One patient $(1.7 \%)$ had a transient paralysis of the sixth cranial nerve. No other cranial neuropathies were observed in our series. As regards endocrinological complications, permanent diabetes insipidus occurred in two patients (3.5\%). No postoperative meningitis occurred in our patient group and none of the procedures was complicated by intraoperative injury of the ICA (Table 4).

\section{Discussion}

The NFPA are benign lesions that may attain large dimensions over time without being detected. This is why $10-40 \%{ }^{(15)}$ of cases of NFPA are diagnosed late and when they are already large enough to be classified as macroadenomas (greater than $1 \mathrm{~cm}$ ). 


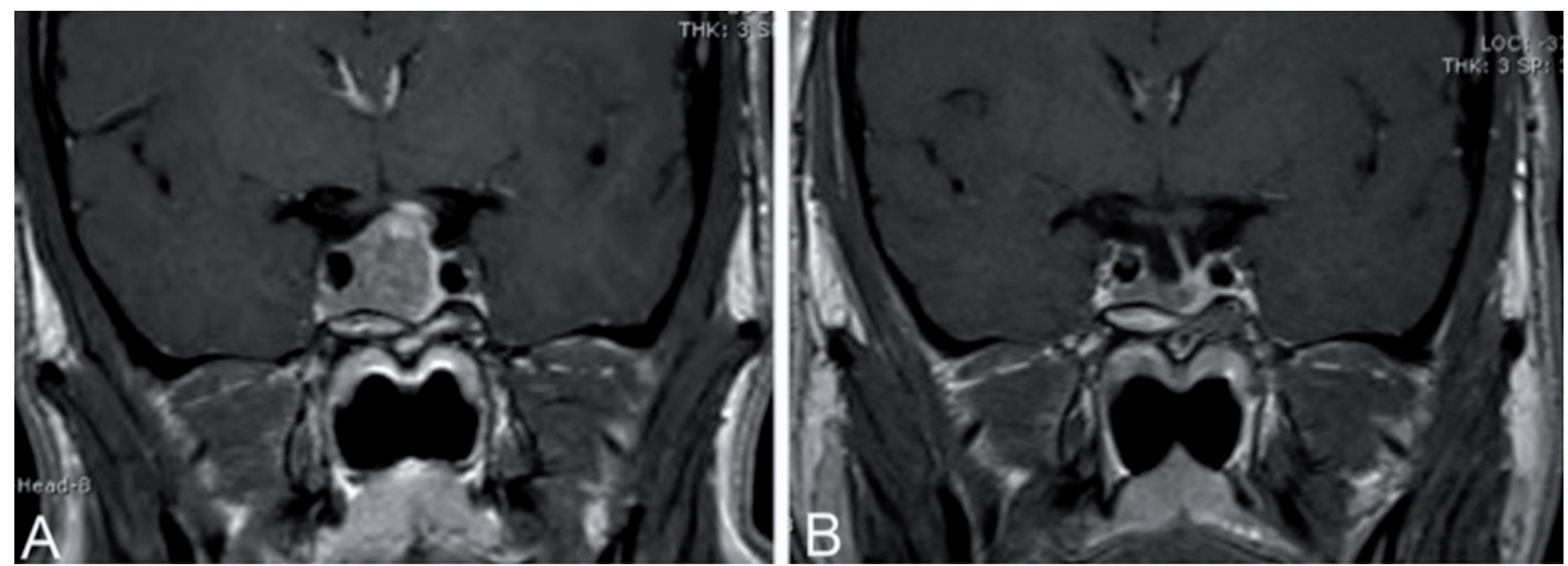

Figure 3. Preoperative contrast-enhanced T1-weighted MRI scan in coronal (A) view, showing a non-functioning pituitary adenoma invading the inferior compartment of the right cavernous sinus. The 1-year postoperative contrast-enhanced T1-weighted MRI scan in coronal (B) view revealed a sub-total resection of the lesion.

When symptoms are present, visual disturbance or headaches are the main complaints.

It is widely accepted that trans-sphenoidal or transacranial surgery can improve visual defects for most patients with these lesions ${ }^{(16-18)}$. Meta-analysis studies have shown that surgery provided a significant improvement in the symptoms of $78 \%$ of patients presenting with VA defects ${ }^{(19)}$. The efficacy and safety of the surgical procedure is also demonstated in our series, where a normalization or an improvement of VA and VF defects were obtained in $81 \%$ of patients, with only one reporting a worsening of VA deficit.

Several studies analyzing the results of endocrinological hypersecretion syndrome in patients with pituitary adenomas have been published. However there are only a few which have focused on pituitary function after surgery for NFPA. The largest, by Nomikos et al., described the endocrinological outcomes from a series of 660 NFPA which were surgically removed using a microscopic technique. Partial anterior hypopituitarism was normalized or improved in $49.7 \%$ of cases, remained stable in $48.9 \%$ and worsened in $1.4 \%{ }^{(20)}$. In our series a normalization or at least improvement of previous hypopituitarism was obtained in $55 \%$ of cases. Notably, no significant differences in endocrinological outcomes were observed between GTR and STR, suggesting that the extent of resection does not have a direct impact on the resulting pituitary function. This aspect is related to the lateral position of the residual tumor, distant from the pituitary gland. Data regarding endocrinological outcomes after endoscopic trans-sphenoidal surgery for NFPA are limited and conflicting. Some authors have reported an improvement in the hormonal profile after surgery ${ }^{(20)}$, while others found no significant improvements ${ }^{(21)}$ or even a decrease in pituitary function ${ }^{(22)}$. A recent study reported improvement or normalization of pituitary function in $56 \%$ of patients who underwent endoscopic transnasal surgery in comparison with only $25 \%$ in the group treated using the traditional microscopic technique ${ }^{(23)}$. These results support the concrete possibility of pituitary function recovery after surgery and indicate that tumor resection should be performed through a conservative and selective technique, in order to preserve as much healthy residual glandular tissue as possible.

As regards the endocrine management of hypopituitarism, the primary approach is hormone-replacement therapy. Each patient's endocrine abnormalities and associated pathologies should be individually evaluated in order to determine the optimum approach. In our series postoperative glucocorticoid replacement was not mandatory but was administered individually based on each patient's postoperative day 2 and 6 serum cortisol levels. In our experience adequate treatment with glucocorticoids is valuable not only during the perioperative course but also in improving patients' overall condition.

The rate of GTR varies with different case-series and even falls to zero when dealing with NFPA with lateral extension and CSI which are the main limitations on complete tumor resection. Dehdashti et al. reported their surgical experience of 111 NFPAs treated through an endoscopic transnasal approach which achieved an overall GTR of $88 \%$, ranging from $97 \%$ of GTR for non-invasive macroadenomas to $0 \%$ in the case of tumors with $\mathrm{CSI}^{(6)}$. Chang et al. obtained subtotal resection in all their cases of NFPA with CSI using the microscopic sub-labial transsphenoidal approach (24). Recently, several studies have reported better results in the treatment of NFPA with CSI using the EEA. Frank et al. reported a series of 35 patients affected by NFPA where $20 \%$ had intraoperative evidence of CSI and yet GTR was achieved in $60 \%$ of cases ${ }^{(25)}$. Cappabianca et al. described a CSI in $23.7 \%$ of 


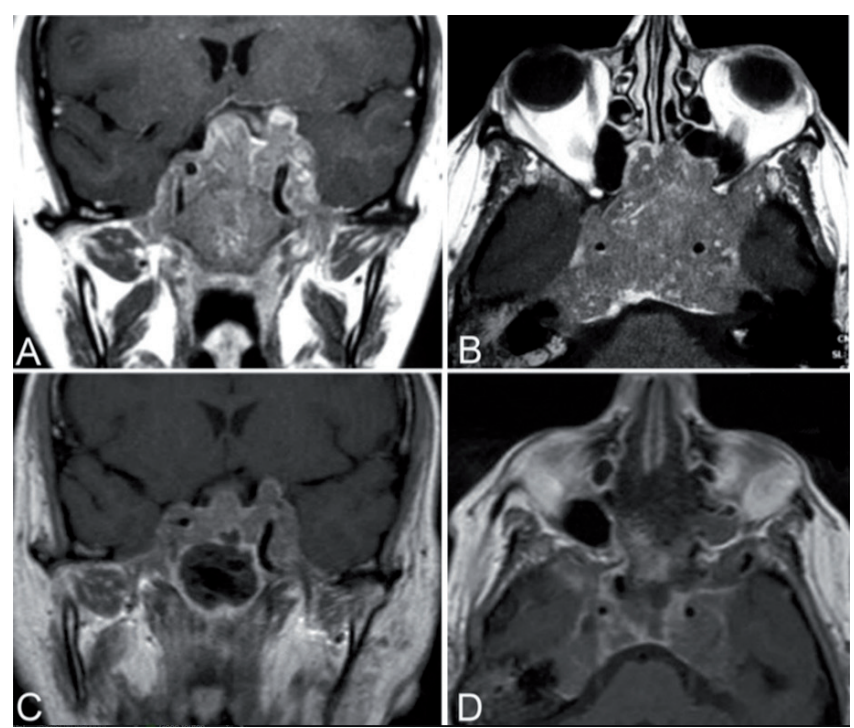

Figure 4. Preoperative contrast-enhanced T1-weighted MRI scan in axial (A) and coronal (B) view, showing a giant non-functioning pituitary adenoma with bilateral invasion of the cavernous sinuses and with complete encasement of both internal carotid arteries. The 3-month postoperative contrast-enhanced T1-weighted MRI scan in axial (C) and coronal (D) view revealed a partial resection of the lesion.

the NFPA treated, obtaining a GTR in $36.8 \%$ of them ${ }^{(5)}$. Paluzzi et al. reported a CSI in $34.5 \%$ of the NFPA they treated, their group including a Knosp grade 2 tumor, and achieved a GTR in $35.4 \%$ of cases ${ }^{(26)}$.

Most recently several experiences in the treatment of giant pituitary adenomas reported variable rates of GTR ranging from $20.7 \%{ }^{(27)}$ to $24 \%{ }^{(28)}$, but no data about CSI and pituitary function were specified.

Data emerging from our series are consistent with these other results: we reported a CSI in $27.3 \%(56 / 205)$ of the NFPA treated, including in this group only the Knosp grade 3 and 4 tumors with concordant radiological and intraoperative findings, and obtained a GTR in $30.3 \%$ of cases and STR in $60.7 \%$.

It is widely accepted that CSI is the main predictive factor for recurrence after surgical resection of NFPAs ${ }^{(21,24,29,30)}$. At the same time, and without providing overconfidence as to future disease control rates, the extent of the resection obtained could also be an important factor in predicting recurrence. An illustration of such predictive difficulty is that Park et al. described a high rate of 19/26 recurrences after GTR while Chang et al. reported 19/307 recurrences after GTR, occurring in six patients treated with surgery and adjuvant radiotherapy and in 13 cases treated with surgery alone. The average recurrence time was respectively 5.3 and 6.9 years ${ }^{(31)}$. Although it is clear that tumor regrowth depends on the extent of the tumor and on the resection obtained, the role of post-operative residual disease in long-term tumor control for non-irradiated patients is still de- bated: some studies have observed only limited rates of tumor regrowth in the presence of residual disease after exclusively surgical treatment ${ }^{(22)}$. Our experience of NFPA with CSI seems to be consistent with this trend, showing long-term tumor control without regrowth/recurrence of tumor in $87.5 \%$ (49/56) of cases after a mean follow-up of 61 months. This is in line with previous studies reporting a long-term control rate ranging from $79.9 \%$ to $90 \%{ }^{(22,30)}$. In our series no recurrences were observed after GTR and only 7/39 showed regrowth of residual tumor after subtotal or partial resection. Nevertheless, extended follow-up beyond five years is recommended because of the possibility of recurrence or residual tumor regrowth up to ten years after treatment ${ }^{(32)}$.

In our series only one patient underwent adjuvant radiotherapy 23 months after surgery. In this case the lesion was considered Knosp grade 4 and its consistency was so hard that the removal of the residual disease involving the cavernous sinus appeared much too risky to be attempted. However, this case was exceptional and overall our long-term data are unable to justify prophylactic adjuvant radiotherapy for residual disease in the field of NFPA. Given the slow growth and the benign behaviour of these tumors, radiation therapy could be recommended only in case of patients with long life expectancy (> 10 years), when the postoperative residual tumor is clinically significant or when the periodic imaging scans reveal a progression of disease which would be impossible to optimally manage with surgical revision.

\section{Complications analysis}

The most frequent complication in pituitary surgery is CSFleak which is reported in between $1.2 \%$ and $6 \%$ of the largest endoscopic case-series ${ }^{(5-8,25,33)}$. The rates of this complication increase significantly in the case of extended approaches reaching $4.5 \%-11 \%$, based on the greater exposure of the sellar dura ${ }^{(4,7,34-39)}$. In our series, post-operative CSF-leak requiring sellar floor reconstruction occurred in 5.3\% of cases. Significantly, from 2008 a vascularized pedicled nasoseptal flap has been used to overlay the skull base reconstruction which has dramatically reduced the rates of CSF-leakage. Nowadays when intraoperative CSF-leak is evident, our sellar floor reconstruction technique routinely uses a pedicled nasoseptal flap placed in overlay fashion, covering the inlay autologous tissue grafts. The outcomes of this technique are encouraging, necessitating no further surgical revision up to now.

Permanent diabetes insipidus was observed in $3.5 \%$ of cases, similar to that described in previous endoscopic endonasal experiences ${ }^{(5-8,25,33)}$. No nasal complications such as post-operative epistaxis, were reported in our study. No lesions of the ICA occurred in our series, thanks to proper anatomical knowledge and extensive surgical experience that also took advantage of the intraoperative use of the Doppler Ultrasound and magnetic neuronavigation system. No permanent cranial neuropathies 
were observed in our series, with only one case of sixth cranial nerve transient palsy. These results are in accordance with our conservative philosophy on the management of CSI, where preservation of the cranial nerves is considered the key endpoint with the extent of resection always subordinate to it.

\section{Limitations}

The study has several limitations, largely related to its retrospective design, that must be considered when interpreting the results. Moreover, the immunophenotype of NFPA is a critical point that needs further investigation. Future studies should analyse histopathological and immunohistochemical markers in order to classify the NFPA in subgroups with different biological behaviours the better to tailor appropriate treatment strategies (40).

\section{Conclusions}

Surgical treatment of NFPA with CSI aims at removal of as great a part of the tumor as possible in order to reduce the mass effect and minimize clinical symptoms for the patient. The extent of tumor removal depends directly on the extent of disease and is strongly linked to the Knosp grading system. Above all, the risks of surgical morbidity when treating benign lesions extending into the CS must be taken into account. The EEA has proven to be a safe and effective procedure without evidence of tumor recurrence or regrowth in $87.5 \%$ (49/56) of patients in the present series after a mean follow-up of five years. On the basis of the data presented in this study we advocate a post-surgical wait-and-see policy in cases of residual tumor. This is in order to prevent any unnecessary potential sequelae of adjuvant radiotherapy for the majority of patients. Further studies based on larger patient cohorts and with assessment of tumor control rates extending beyond five years are required to validate our data.

\section{Author contributions}

Study concept and design: FF; Acquisition of data: FF; Analysis and interpretation of data: FF, MTZ; Drafting of the manuscript: FF, MTZ, PB; Critical revision of the manuscript for important intellectual content: PC, MB, FRC, DL; Statistical analysis: FF, MTZ; Administrative, technical, material supportand study supervision: PC, DL.

\section{Conflict of interest}

All the authors certify that they have no conflict of interest or financial relationship with any entity mentioned in the paper. No sponsors or grants are involved in the paper.

\section{References}

1. Asa SL, Kovacs K. Clinically non-functioning human pituitary adenomas. Can J Neuro Sci. 1992;19:228-235.

2. Greeman Y, Stern N. Non-functioning pituitary adenomas. Best Pract Res Clin Endocrinol Metab. 2009;23:625-638.

3. William F, Young, Jr. Clinically Nonfunctioning Pituitary Adenomas. In: Thapar K, Kovacs K, Scheithauer BW, Lloyd RV, (eds): Diagnosis and Management of Pituitary Tumors. New Jersey: Humana Press; 2001; 343-351.

4. Couldwell WT, Weiss MH, Rabb C, Liu JK, Apfelbaum RI, Fukushima T. Variations on the standard transsphenoidal approach to the sellar region, with emphasis on the estende approaches and parasellar approaches: surgical experience in 105 cases. Neurosurgery 2004;55:539-547.

5. Cappabianca P, Cavallo LM, Colao A, de Divitiis E. Surgical complications associated with the endoscopic endonasal transsphenoidal approach for pituitary adenomas. J Neurosurg. 2002;97:293-298.

6. Dehdashti AR, Ganna A, Karabatsou K, Gentili F. Pure endoscopic endonasal approach for pituitary adenomas: early surgical results in 200 patients and comparison with previous microsurgical series. Neurosurgery 2008;62:1006-1015.

7. Frank G, Pasquini E, Farneti G, et al. The endoscopic versus the traditional approach in pituitary surgery. Neuroendocrinology 2006:83:240-248.

8. Gondim JA, Almeida JP, Albuquerque LA, et al. Endoscopic endonasal approach for pituitary adenoma: surgical complications in 301 patients. Pituitary 2011;14:174-183.

9. Knosp E, Steiner E, Kitz K, Matula C. Pituitary Adenomas with Invasion of the Cavernous Sinus Space: A Magnetic Resonance Imaging Classification Compared with Surgical Findings. Neurosurgery 1993;33:610-617.

10. Castelnuovo P, Pistochini A, Locatelli D. Different surgical approaches to the sellar region: focusing on the "two nostrils four hands technique." Rhinology 2006;44:2-7.

11. Locatelli D, Canevari FR, Acchiardi I, Castelnuovo P. The endoscopic diving technique in pituitary and cranial base surgery: technical note. Neurosurgery 2010;66(2):E400-401.

12. Castelnuovo P, Mauri S, Locatelli D, Emanuelli E, Delù G, Giulio GD. Endoscopic repair of cerebrospinal fluid rhinorrhea: learning from our failures. Am J Rhinol. 2001;15:333-342.

13. Castelnuovo PG, Delù G, Locatelli $D$, et al. Endonasal endoscopic duraplasty: our experience. Skull Base 2006;16:19-24.

14. Hadad G, Bassagasteguy L, Carrau RL, et al. A novel reconstructive technique after endoscopic expanded endonasal approaches: vascular pedicle nasoseptal flap. Laryngoscope 2006;116:1882-1886.

15. Dekkers OM, Hammer S, de Keizer RJ, et al. The natural course of non-functioning pituitary macroadenomas. Eur J Endocrinol 2007:156:217-224.

16. de Paiva Neto MA, Vandergrift A, Fatemi N et al. Endonasal transsphenoidal surgery and multimodality treatment for giant pituitary adenomas. Clinical Endocrinol (Oxf) 2010;72:512-519.

17. Mortini P, Barzaghi R, Losa M, Boari N, Giovanelli M. Surgical treatment of giant pituitary adenomas: strategies and results in a series of 95 consecutive patients. Neurosurgery 2007;60:993-1002.

18. Nielsen EH, Lindholm J, Laurberg P, et al: Nonfunctioning pituitary adenoma: incidence, causes of death and quality of life in relation to pituitary function. Pituitary 2007; 10:67-73.

19. Murad MH, Fernàndez-Balsells MM, Barwise A, et al. Outcomes of surgical treatment for nonfunctioning pituitary adenomas: a systematic review and meta-analysis. Clin Endocrinol (Oxf) 2010;73:777-791.

20. Nomikos P, Ladar C, Fahlbusch R, Buchfelder M. Impact of primary surgery on pituitary function in patients with non-functioning pituitary adenomas--a study on 721 patients. Acta Neurochir (Wien) 2004;146:27-35.

21. Comptois R, Beauregard $H$, Somma M, Serri $\mathrm{O}$, Aris-Jilwan N, Hardy J. The clinical and 
endocrine outcome to trans-sphenoidal microsurgery of nonsecreting pituitary adenomas. Cancer 1991;68:860-866.

22. Dekkers OM, Pereira AM, Roelfsema F, et al. Observation alone after transsphenoidal surgery for nonfunctioning pituitary macroadenoma. J Clin Endocrinol Metab 2006 91:1796-1801.

23. Messerer M, De Battista JC, Raverot G, et al Evidence of improved surgical outcome following endoscopy for nonfunctioning pituitary adenoma removal. Neurosurg Focus 2011 30:E11.

24. Chang EF, Zada G, Kim S, et al. Long-term recurrence and mortality after surgery and adjuvant radiotherapy for nonfunctional pituitary adenomas. J Neurosurg 2008 108:736-745.

25. Frank G, Pasquini E. Endoscopic endonasal cavernous sinus surgery, with special reference to pituitary adenomas. Front Horm Res 2006 34:64-82.

26. Paluzzi A, Fernandez-Miranda JC, Tonya Stefko S, Challinor S, Snyderman $\mathrm{CH}$ Gardner PA. Endoscopic endonasal approach for pituitary adenomas: a series of 555 patients. Pituitary 2014;17(4):307-319.

27. Cusimano MD, Kan P, Nassiri F, et al. Outcomes of surgically treated giant pituitary tumours. Can J Neurol Sci. 2012;39(4):446-457.

28. Juraschka K, Khan OH, Godoy BL, et al. Endoscopic endonasal transsphenoidal approach to large and giant pituitary adenomas: institutional experience and predictors of extent of resection. J Neurosurg. 2014;121(1):75-83.

29. Greenman Y, Ouaknine G, Veshchev
I, Reider-Groswasser II, Segev Y, Stern $\mathrm{N}$. Postoperative surveillance of clinically nonfunctioning pituitary macroadenomas: markers of tumour quiescence and regrowth. Clin Endocrinol 2003 (Oxf) 58:763-769, 2003

30. Losa M, Mortini P, Barzaghi R, et al. Early results of surgery in patients with nonfunctioning pituitary adenoma and analysis of the risk of tumor recurrence. J Neurosurg 2008;108:525-532.

31. Park P, Chandler WF, Barkan AL, et al. The role of radiation therapy after surgical resection of nonfunctional pituitary macroadenomas. Neurosurgery 2004;55:100-106.

32. Dekkers OM, Pereira AM, Romijn JA Treatment and follow-up of clinically nonfunctioning pituitary macroadenomas. J Clin Endocrinol Metab 2008;93:3717-3726.

33. Jho HD. Endoscopic transsphenoidal surgery. J Neurooncol 2001;54:187-195.

34. Ceylan S, Koc K, Anik I. Endoscopic endonasal transsphenoidal approach for pituitary adenomas invading the cavernous sinus. $J$ Neurosurg 2010;112:99-107.

35. Dumont AS, Kanter AS, Jane JA Jr, Laws ER Jr. Extended transsphenoidal approach. Front Horm Res 2006:34:29-45.

36. Kitano M, Taneda M. Extended transsphenoidal approach with submucosal posterior ethmoidectomy for parasellar tumors. Technical note. J Neurosurg 2001;94:9991004.

37. Kitano M, Taneda M, Shimono T, Nakao Y. Extended transsphenoidal approach for surgical management of pituitary adenomas invading the cavernous sinus. J Neurosurg 2008;108:26-36
38. Sciarretta $\vee$, Mazzatenta D, Ciarpaglini $R$, Pasquini E, Farneti G, Frank G. Surgical repair of persisting CSF leaks following standard or extended endoscopic transsphenoidal surgery for pituitary tumor. Minim Invasive Neurosurg 2010;53:55-59.

39. Zhao B, Wei YK, Li GL, et al. Extended transsphenoidal approach for pituitary adenomas invading the anterior cranial base, cavernous sinus, and clivus: a single-center experience with 126 consecutive cases. J Neurosurg 2010;112:108-117

40. Bradley KJ, Wass JA, Turner HE. Nonfunctioning pituitary adenomas with positive immunoreactivity for ACTH behave more aggressively than ACTH immunonegative tumours but do not recur more frequently. Clin Endocrinol (Oxf) 2003;58(1):5964.

\section{Fabio Ferreli, MD}

Department of Otorhinolaryngology

University of Insubria Varese

Via Guicciardini 9

Varese

Italy

Tel: +39-332-278426

Fax: +39-0332-278945

E-mail:fabio_ferreli@yahoo.it

\section{CORRIGENDUM}

In the paper by Wofford et al., 2015, Rhinology 53(1):41-49 the Acknowledgements contained a wrong referral to a grant number. The correct grant number is T32 DC005360. This corrigendum is to indicate that fact. 